\title{
Acute kidney injury after partial nephrectomy: transient or permanent kidney damage? - Impact on long-term renal function
}

\author{
Giuseppe Rosiello, Umberto Capitanio, Alessandro Larcher \\ Department of Urology and Division of Experimental Oncology, URI, Urological Research Institute, IRCCS San Raffaele Scientific Institute, Milan, \\ Italy \\ Correspondence to: Giuseppe Rosiello, MD. Department of Urology and Division of Experimental Oncology, URI, Urological Research Institute, \\ IRCCS San Raffaele Scientific Institute, Olgettina 60, Milan 20132, MI, Lombardia, Italy. Email: giusepperosiello@hotmail.it. \\ Provenance: This is an invited article commissioned by the Section Editor Dr. Xiao Li (Department of Urology, Jiangsu Cancer Hospital, Jiangsu \\ Institute of Cancer Research, Nanjing Medical University Affiliated Cancer Hospital, Nanjing, China). \\ Comment on: Bravi CA, Vertosick E, Benfante N, et al. Impact of Acute Kidney Injury and Its Duration on Long-term Renal Function After Partial \\ Nephrectomy. Eur Urol 2019;76:398-403.
}

Submitted Sep 18, 2019. Accepted for publication Sep 29, 2019.

doi: 10.21037/atm.2019.09.156

View this article at: http://dx.doi.org/10.21037/atm.2019.09.156

Partial nephrectomy represents the gold standard treatment for cT1 renal masses $(1,2)$. Indeed, several studies demonstrated the superiority of nephron-sparing approach relative to radical approach, with respect to long-term renal function $(3,4)$. Despite the lack of experimental data, multiple observational investigations described important clinical consequences related to such functional benefit. For example, the clinical sequelae of such benefit range from lower cardiovascular morbidity to potential survival benefit (5-7).

These observations, together with a stage migration phenomenon towards a less aggressive disease, prompted an increase of partial nephrectomy use over time (8-10).

The preservation of renal function after partial nephrectomy represents one of the most important goal of the surgery and it is invariably regarded as a marker of surgical quality $(11,12)$. However, the core of the relationship between superior renal function following oncologic surgery and the potential lower cardiovascular events and improved survival are not fully elucidated.

Multiple reports demonstrated that worse renal function after surgery impacts morbidity and mortality (13-15). Chawla et al. (15) reviewed the interconnection between acute kidney injuries (AKI), defined according to the RIFLE (risk-injury-failure-loss-end stage) criteria as more than 25\% reduction in baseline eGFR (estimated glomerular filtrate rate) or $>1.5$-fold increase in baseline creatinine, both at discharge from hospital, and chronic kidney disease (CKD), which is invariably associated with poor survival outcomes $(16,17)$. Moreover, several findings suggest that AKI not only is directly linked to the progression of CKD, but the increased severity of AKI, as well as multiple episodes of AKI are associated with the newly onset CKD (15). A systematic review and meta-analysis investigated the association between the duration of AKI and survival in hospitalized patients (18). Specifically, Mehta et al. (18) reported an increased risk of both cardiovascular events and incident CKD according to duration of AKI $(\leq 2,3-6$ and $\geq 7$ days). Moreover, the risk of long-term mortality was higher for longer AKI. Pooled risk ratios reported for longterm mortality according to duration of AKI were 1.42, 1.92 and 2.28 for $\leq 2,3-6$ and $\geq 7$ days of AKI, respectively. A similar relationship has been shown for cardiovascular events and incident CKD. In light of these findings, it may be postulated that to prevent prolonged AKI is essential in the post-operative management of patients undergoing nephron-sparing surgery, as partial nephrectomy invariably involves kidney manipulation and as such, it implies a detrimental effect on renal function per-se.

Does the occurrence of AKI invariably lead to CKD? The available evidence $(19,20)$ did not demonstrate a direct correlation between severity of AKI and long-term renal function decline. Moreover, the magnitude of the effect of AKI on long-term functional outcomes is unknown, in the context of partial nephrectomy candidates. 
In this context, Bravi et al. (21) provided a pivotal contribute for a better understanding of the role of AKI after partial nephrectomy. Investigators relied on a singleinstitution database and identified 1,893 patients, who underwent partial nephrectomy between 1989 and 2018 for cT1 renal mass. To assess the relationship between AKI and long-term renal function, the Authors identified 3 outcomes of interest: 1. recovery of at least $90 \%$ of baseline function 1 year after partial nephrectomy; 2. percentage change of 1-year renal function compared with baseline function; 3 . CKD upstaging after surgery.

Their results showed several important observations. First, it is noteworthy that $20 \%$ of patients in their cohort experienced AKI after partial nephrectomy. This figure is lower than that recorded in other studies $(22,23)$. For example, Rajan et al. (22) reported up to $40 \%$ of patients, who experienced AKI after nephron-sparing surgery. Despite lower proportion of AKI has been reported by Bravi et al. (21) compared with Rajan et al. (22), this observation demonstrate that AKI is a post-operative complication, which can be experienced in a considerably high proportion of patients (from $11 \%$ to $40 \%)(21,22,24)$. Second, postoperative AKI has been associated with worse functional outcomes at 1-year follow-up. Specifically, patients, who experienced AKI after surgery, demonstrated worse recovery of pre-operative eGFR ( $30 \%$ vs. $61 \%$ ), higher rates of CKD upstaging ( $51 \%$ vs. $23 \%$ ) and worse changes of eGFR compared to baseline $(-17 \%$ vs. $-1 \%)$, at 1 -year followup. In light of these findings, two important considerations may be derived. First, these results clarify the link between $\mathrm{AKI}$ and renal function detriment after surgery. Second, it is noteworthy that partial nephrectomy has an intrinsic risk of functional harm, as only $61 \%$ of patients, who did not experience AKI, recovered more than $90 \%$ of baseline renal function after surgery and that $23 \%$ of them experienced CKD upstaging. Third, a direct correlation between duration of AKI and all the three outcomes of interest has been shown.

Interestingly, it is particularly impressive that, despite a linear relationship between prolonged AKI and worse functional outcomes (21), the most striking differences depended on the duration of AKI, particularly after the third day of AKI. For instance, $-22.4 v s .-11.3 v s .-9.3 \%$ of change from baseline eGFR was reported after, respectively, $\geq 4$ vs. 2-3 vs. 1 day of AKI, relative to no AKI. Similarly, the probability of recovering $90 \%$ of eGFR at baseline was $8 \%$ vs. $30 \%$ vs. $40 \%$ in case of $\geq 4 v s .2-3$ vs. 1 days of AKI, respectively. Finally, $\geq 4$ vs. $2-3 v s .1$ day of AKI were associated with 6-fold vs. 3-fold vs. 2-fold higher risk of
CKD upstaging, relative to no AKI.

Taken together, these findings not only demonstrate an essential influence of post-operative AKI on long-term renal function, but also highlight the importance of the duration of AKI. Surgeons should take into account that partial nephrectomy has an intrinsic risk of worse renal function after surgery. Moreover, in presence of AKI, a protracted AKI over three days should be avoided, since worse functional outcomes have been reported after this day-threshold. For example, control of fluid resuscitation in volume depletion, avoiding volume overload, targeting a blood glucose level at least below $180 \mathrm{mg} / \mathrm{dL}(10 \mathrm{mmol} / \mathrm{L})$ for the prevention of hyperglycaemic kidney damage and adequate nutritional support may represent preventative measures aimed at preventing longer AKI (25).

On the view of these findings, it is of note that Martini et al. (26) recently developed a nomogram for the early identification of patients, who are at a high risk of experiencing significant eGFR reduction from their baseline eGFR (27). They found that the presence of AKI in the context of CKD is associated with 4-fold higher risk of significant eGFR reduction, relative to patients with normal renal function. Moreover, the inclusion of AKI in the model conferred an increase in c-index from $75 \%$ to $76 \%$. Additionally, the net benefit originating from the use of the model predicting eGFR reduction was significantly higher, when AKI was included among predicting variables. These findings confirm the role that postoperative AKI plays in the prediction of eGFR reduction and support the use of this model for patient counseling.

Several studies discussed potential determinants of AKI after partial nephrectomy. Specifically, Zhang et al. (28) analyzed data of 83 patients with solitary kidney, who underwent nephron-sparing surgery for renal masses, showing that the duration of ischemia was invariably associated with higher risk of AKI (OR: 1.06; $\mathrm{P}=0.02$ ). This observation is consistent with the results provided by Thompson et al. (29), who demonstrated that warm ischemia longer than 20 mins and cold ischemia longer than 35 mins are associated with higher incidence of AKI (24.4\% vs. $6.4 \%$ and $32.0 \%$ vs. $13.0 \%$, respectively). Similarly, Lane et al. (30) showed that the risk of AKI after partial nephrectomy was increased by $3 \%$ per percentage of parenchyma preserved $(\mathrm{P}<0.001)(31,32)$, and by $1 \%$ per minute increase of ischemia $(\mathrm{P}<0.06)$. Finally, Rajan et al. (22) analyzed the role of several variables on the risk of AKI after partial nephrectomy. Interestingly, the Authors found that preoperative hypertension, as well as use of 
ACE inhibitors, longer ischemia time and cold ischemia predicted a drop in postoperative eGFR. Moreover, Rajan et al. (22) also demonstrated that the use of robotic approach, compared to either open or laparoscopic approaches, is associated with a lower decrease of postoperative eGFR (33). These findings are also confirmed by Larcher et al. (34), who demonstrated that, compared to open surgery, robot-assisted partial nephrectomy is associated with similar functional outcomes regardless of tumor complexity and patient comorbidities.

In conclusion, the presence of AKI after partial nephrectomy should not be underestimated, since this relatively common complication affects long-term renal function and, consequently, morbidity and mortality of partial nephrectomy candidates. Worse renal function after surgery may result in faster long-term renal function detrimental until the onset of CKD, as well as in CKD upstaging, which has been associated with higher rate of cardiovascular events and thus, higher mortality $(6,7)$. In this context, the results provided by Bravi et al. (21) afforded a key contribute to current literature, demonstrating that AKI not only is an important predictor of renal function recovery over time, but they also demonstrated a linear correlation between duration of AKI and worsening of function after partial nephrectomy. Many efforts should be made to prevent AKI, especially in patients at high risk of developing this complication. Additionally, in presence of AKI, preventative measures aimed at reducing its extension over 3 days should be adopted, since faster worsening of renal function has been demonstrated after this "day-threshold".

\section{Acknowledgments}

None.

\section{Footnote}

Conflicts of Interest: The authors have no conflicts of interest to declare.

Ethical Statement: The authors are accountable for all aspects of the work in ensuring that questions related to the accuracy or integrity of any part of the work are appropriately investigated and resolved.

\section{References}

1. Motzer RJ, Jonasch E, Agarwal N, et al. Kidney Cancer,
Version 2.2017, NCCN Clinical Practice Guidelines in Oncology. J Natl Compr Canc Netw 2017;15:804-34.

2. Ljungberg B, Bensalah K, Canfield S, et al. EAU Guidelines on Renal Cell Carcinoma: 2014 Update. Eur Urol 2015;67:913-24.

3. Leppert JT, Lamberts RW, Thomas I-C, et al. Incident CKD after Radical or Partial Nephrectomy. J Am Soc Nephrol 2018;29:207-16.

4. Wang Z, Wang G, Xia Q, et al. Partial nephrectomy vs. radical nephrectomy for renal tumors: A meta-analysis of renal function and cardiovascular outcomes. Urol Oncol 2016;34:533.e11-533.e19.

5. Larcher A, Capitanio U, Terrone C, et al. Elective Nephron Sparing Surgery Decreases Other Cause Mortality Relative to Radical Nephrectomy Only in Specific Subgroups of Patients with Renal Cell Carcinoma. J Urol 2016;196:1008-13.

6. Larcher A, Sun M, Dell'Oglio P, et al. Mortality, morbidity and healthcare expenditures after local tumour ablation or partial nephrectomy for T1A kidney cancer. Eur J Surg Oncol 2017;43:815-22.

7. Capitanio U, Terrone C, Antonelli A, et al. Nephronsparing Techniques Independently Decrease the Risk of Cardiovascular Events Relative to Radical Nephrectomy in Patients with a T1a-T1b Renal Mass and Normal Preoperative Renal Function. Eur Urol 2015;67:683-9.

8. Mir MC, Capitanio U, Bertolo R, et al. Role of Active Surveillance for Localized Small Renal Masses. Eur Urol Oncol 2018;1:177-87.

9. Chow W-H, Devesa SS. Contemporary Epidemiology of Renal Cell Cancer: Cancer J 2008;14:288-301.

10. Patel HD, Gupta M, Joice GA, et al. Clinical Stage Migration and Survival for Renal Cell Carcinoma in the United States. Eur Urol Oncol 2019;2:343-8.

11. Larcher A, De Naeyer G, Turri F, et al. The ERUS Curriculum for Robot-assisted Partial Nephrectomy: Structure Definition and Pilot Clinical Validation. Eur Urol 2019;75:1023-31.

12. Larcher A, Muttin F, Peyronnet B, et al. The Learning Curve for Robot-assisted Partial Nephrectomy: Impact of Surgical Experience on Perioperative Outcomes. Eur Urol 2019;75:253-6.

13. Huang WC, Elkin EB, Levey AS, et al. Partial Nephrectomy Versus Radical Nephrectomy in Patients With Small Renal Tumors-Is There a Difference in Mortality and Cardiovascular Outcomes? J Urol 2009;181:55-61.

14. Coca SG, King JT, Rosenthal RA, et al. The duration 
of postoperative acute kidney injury is an additional parameter predicting long-term survival in diabetic veterans. Kidney Int 2010;78:926-33.

15. Chawla LS, Eggers PW, Star RA, et al. Acute Kidney Injury and Chronic Kidney Disease as Interconnected Syndromes. N Engl J Med 2014;371:58-66.

16. Levey AS, de Jong PE, Coresh J, et al. The definition, classification, and prognosis of chronic kidney disease: a KDIGO Controversies Conference report. Kidney Int 2011;80:17-28.

17. Webster AC, Nagler EV, Morton RL, et al. Chronic Kidney Disease. Lancet 2017;389:1238-52.

18. Mehta S, Chauhan K, Patel A, et al. The prognostic importance of duration of AKI: a systematic review and meta-analysis. BMC Nephrol 2018;19:91.

19. Kawamura N, Yokoyama M, Tanaka H, et al. Acute kidney injury and intermediate-term renal function after clampless partial nephrectomy. Int J Urol 2019;26:113-8.

20. Zabell J, Isharwal S, Dong W, et al. Acute Kidney Injury after Partial Nephrectomy of Solitary Kidneys: Impact on Long-Term Stability of Renal Function. J Urol 2018;200:1295-301.

21. Bravi CA, Vertosick E, Benfante N, et al. Impact of Acute Kidney Injury and Its Duration on Long-term Renal Function After Partial Nephrectomy. Eur Urol 2019;76:398-403.

22. Rajan S, Babazade R, Govindarajan SR, et al. Perioperative factors associated with acute kidney injury after partial nephrectomy. Br J Anaesth 2016;116:70-6.

23. Ercole CE, Velet L, Zhang Z, et al. Functional recovery from extended warm ischemia associated with partial nephrectomy. J Urol 2015;193:e794.

24. Campbell SC, Novick AC, Streem SB, et al. Complications of Nephron Sparing Surgery for Renal Tumors. J Urol 1994;151:1177-80.

25. Joannidis M, Druml W, Forni LG, et al. Prevention of acute kidney injury and protection of renal function in the intensive care unit: update 2017. Intensive Care Med

Cite this article as: Rosiello G, Capitanio U, Larcher A. Acute kidney injury after partial nephrectomy: transient or permanent kidney damage?-Impact on long-term renal function. Ann Transl Med 2019;7(Suppl 8):S317. doi: 10.21037/ atm.2019.09.156
2017;43:730-49.

26. Martini A, Cumarasamy S, Beksac AT, et al. A Nomogram to Predict Significant Estimated Glomerular Filtration Rate Reduction After Robotic Partial Nephrectomy. Eur Urol 2018;74:833-9.

27. Bertolo R, Garisto J, Li J, Dagenais J, et al. Development and Internal Validation of a Nomogram for Predicting Renal Function after Partial Nephrectomy. Eur Urol Oncol 2019;2:106-9.

28. Zhang Z, Zhao J, Dong W, et al. Acute Kidney Injury after Partial Nephrectomy: Role of Parenchymal Mass Reduction and Ischemia and Impact on Subsequent Functional Recovery. Eur Urol 2016;69:745-52.

29. Thompson RH, Frank I, Lohse CM, et al. The Impact of Ischemia Time During Open Nephron Sparing Surgery on Solitary Kidneys: A Multi-Institutional Study. J Urol 2007;177:471-6.

30. Lane BR, Babineau DC, Poggio ED, et al. Factors Predicting Renal Functional Outcome After Partial Nephrectomy. J Urol 2008;180:2363-8.

31. Porpiglia F, Amparore D, Checcucci E, et al. Parenchymal Mass Preserved after Partial Nephrectomy and "Global Renal Damage": Two Faces of the Same Coin. Eur Urol Oncol 2019;2:104-5.

32. Wu J, Suk-Ouichai C, Dong W, et al. Vascularized Parenchymal Mass Preserved with Partial Nephrectomy: Functional Impact and Predictive Factors. Eur Urol Oncol 2019;2:97-103.

33. Peyronnet B, Khene Z-E, Mathieu R, et al. Robot-assisted Versus Open Partial Nephrectomy: Do We Really Need More Evidence To End the Debate? Eur Urol Oncol 2018;1:69-70.

34. Larcher A, Capitanio U, De Naeyer G, et al. Is Robotassisted Surgery Contraindicated in the Case of Partial Nephrectomy for Complex Tumours or Relevant Comorbidities? A Comparative Analysis of Morbidity, Renal Function, and Oncologic Outcomes. Eur Urol Oncol 2018;1:61-8. 